\title{
DISERTACIONES
}

Para citar este artículo: Ponce Capdeville, B., \& Medina Ávila, V. (2019). La voz de Dios: características en el cine de Hollywood. Anuario Electrónico de Estudios en Comunicación Social "Disertaciones", 12(2), 179-196. Doi: http://dx.doi.org/10.12804/revistas.urosario.edu.co/disertaciones/a.6407

\section{LA VOZ DE DIOS: CARACTERÍSTICAS EN EL CINE DE HOLLYWOOD}

\section{The Voice of God: Characteristics in Hollywood Cinema}

\section{A voz de Deus: características no cinema de Hollywood}

\author{
Berenice Ponce Capdeville, Universidad Iberoamericana (México) \\ bponcecapdeville@gmail.com
}

Virginia Medina Ávila*, Universidad Nacional Autónoma de México (México)

virginiamedinaavila9@gmail.com

\section{Recibido: 15 de enero de 2018}

Aceptado: 16 de mayo de 2018

\section{RESUMEN}

El propósito de esta comunicación es analizar cómo se representa la voz de Dios en el cine y toma como punto de referencia películas de Hollywood.

El interés es contribuir al estudio de la voz, más allá de los círculos de los especialistas foniatras y lingüistas, como objeto significante, personaje-voz (Chion, 2004, p. 29) en la narrativa cinematográfica; así como proponer una metodología de análisis para pensar la voz en el cine, desde una perspectiva cualitativa. El modelo elegido para analizar el lenguaje sonoro, y en particular describir la voz y los diferentes signos sonoros que poseen significado narrativo en el filme, parte del enfoque narratológico posclásico propuesto por David Herman (1999) y se asienta en la audionarratología, su más reciente disciplina.

* Producto beca PASPA, UNAM (marzo-julio, 2015) 


\section{DISERTACIONES}

ESTUDIOS

El papel del sonido en la comunicación: contribución, funciones y efectos

ISSN: $1856-9536$

Doi: http://dx.doi.org/10.12804/revistas.urosario.edu.co/disertaciones/a.6407

Volumen 12, Número 2 / Julio-diciembre 2019

Versión PDF para imprimir desde

http://revistas.urosario.edu.co/index.php/disertaciones

De esta manera, el análisis realizado en el lenguaje sonoro utilizado para representar la voz de Dios en doce películas del cine hollywoodense bajo variables cualitativas permite concluir que, a pesar de haber detectado ciertos matices diferenciados, existen convenciones mantenidas a través de los años cuando de la representación de la voz de Dios en el cine de Hollywood se trata.

Palabras clave: voz, cine, personaje-voz, lenguaje sonoro, audionarratología, voz de Dios.

\section{ABSTRACT}

The purpose of this communication is to analyze how the voice of God is represented in the cinema and takes as a point of reference in Hollywood films.

The interest is to contribute to the study of the voice beyond the circles of phonetic and linguistic specialists as a significant object and a character-voice (Chion, 2004, p. 29) in the cinematographic narrative as well as propose a methodology of analysis to think the voice in the cinema, from a qualitative perspective. The chosen model to analyze the sound language and, in particular, to describe the voice and the different sound signs that have narrative meaning in the film starts from the post-classic narratological approach proposed by David Herman (1999) and is based on audio-narratology his most recent discipline.

In this way, the analysis carried out in the sonorous language used to represent the voice of God in twelve Hollywood films under qualitative variables allowed us to conclude that in spite of having detected different nuances there are conventions maintained through the years when its representation in Hollywood cinema is involved.

Keywords: Voice, cinema, character-voice, sound language, audio-narratology, voice of God.

\section{RESUMO}

O propósito desta comunicação é analisar como se representa a voz de Deus no cinema e tem como ponto de referência filmes de Hollywood.

O interesse e contribuir ao estudo da voz, para além do círculo dos especialistas, foniatras e linguistas, como objeto significante, personagem-voz (Chion, 2004, p. 29) na narrativa cinematográfica, assim como propor uma metodologia de análise para pensar a voz no cinema, desde uma perspectiva qualitativa. O modelo elegido para analisar a linguagem sonora, e particularmente descrever a voz e os diferentes signos sonoros que possuem significado narrativo no filme, parte do enfoque narratológico pós-clássico proposto por David Herman (1999) e se assenta na audionarratologia sua mais recente disciplina.

Desta maneira, a análise realizada na linguagem sonora utilizada para representar a voz de Deus em doze filmes do cinema hollywoodiano sob variáveis qualitativas, permite concluir que apesar de ter detectado certos matizes diferenciados, existem convenções mantidas através dos anos em quanto à representação da voz de Deus no cinema de Hollywood se refere.

Palavras-chave: voz, cinema, personagem-voz, linguagem sonora, audionarratologia, voz de Deus. 


\section{DISERTACIONES}

ESTUDIOS

El papel del sonido en la comunicación: contribución, funciones y efectos

ISSN: 1856-9536

Doi: http://dx.doi.org/10.12804/revistas.urosario.edu.co/disertaciones/a.6407

Volumen 12, Número 2 / Julio-diciembre 2019

Versión PDF para imprimir desde

http://revistas.urosario.edu.co/index.php/disertaciones

Vivimos rodeados por un universo de voces y sonidos. En Listening, Barthes resalta nuestra experiencia diaria inmersa en sonidos ligados a nuestro acontecer cotidiano. Por ejemplo, al estar en casa simplemente integramos esos sonidos en una especie de sinfonía doméstica compuesta por voces, ruidos de cocina, sonidos de diferentes puertas, gorgoteo de tuberías y murmullos del exterior, entre otros (1976, p. 218).

De manera similar, los sonidos escuchados al disfrutar de un filme se engarzan unos con otros para dar una sensación de totalidad. Tal sensación es indispensable para conseguir la credibilidad y la verosimilitud del mundo creado.

Sin embargo, la mayor parte de la crítica y de la teoría cinematográfica tienden a subordinar lo sonoro. Es frecuente el empleo de términos y conceptos visuales para referirse a cuestiones sonoras. Se habla de sonidos altos o bajos, del color tímbrico de un arreglo musical, de la imagen estereofónica, de voces fuera de cuadro, en off, de sonidos limpios y transparentes, o de su contrario, sucios y opacos, por citar algunos ejemplos.

Tanto los estudiosos del sonido como técnicos e ingenieros muestran la misma tendencia: conceptualizan lo sonoro de una manera que solo tiene sentido para la imagen. Larson (2015) resume esta tendencia de la siguiente manera: "Estamos conscientes de que hablamos sobre sonido, pero en realidad estamos pensando en la imagen visual de la fuente sonora" (p. 148).

Para revertir esta corriente y contribuir a las investigaciones acerca de los componentes del lenguaje sonoro, convendría el empleo de conceptos relativos al mundo del sonido y, desde luego, el desarrollo de nuevos términos para describir y analizar de forma más precisa el objeto de estudio. Las pruebas de su relevancia en la construcción del lenguaje cinematográfico subrayan esta necesidad.

En el caso específico de la voz, hay evidencias claras de su fascinante poder y Don LaFontaine es una de ellas. Conocido con el sobrenombre 'The Voice of God', LaFontaine (1940-2008) alcanzó la fama como la voz en off de la mayoría de avances o trailers de las películas de Hollywood. Tal fue la confianza de la industria en el potencial expresivo de su voz, ligada a su incidencia sobre el inconsciente del público cinéfilo y, por lo tanto, en relación con su capacidad de venta del producto.

Si las cualidades sonoras de la voz tienen semejante trascendencia, entonces, ¿cómo pensar la voz? Concretamente, ¿cómo pensar la voz de Dios en las películas de Hollywood? En especial, cuando la voz desempeña un papel crucial, como el de representar, mediante su fuerza expresiva, a la divinidad.

\section{Aproximaciones al estudio del lenguaje sonoro en el cine}

La preponderancia de lo visual en las investigaciones sobre los medios audiovisuales en detrimento del lenguaje sonoro hizo considerar otras perspectivas para llevar a cabo este análisis. Por ello, decidimos integrar conceptos y métodos de la audionarratología para analizar la relación entre sonido y narrativa en el cine.

Si bien el enfoque narratológico ha sido aplicado en investigaciones sobre el lenguaje cinematográfico, como podemos constatar en los estudios de Chatman (1978) y Bordwell (2004), al incluir categorías como: iluminación, ángulos de la cámara, encuadres, gesto y diálogo, dicho enfoque no se ha hecho extensivo a las categorías particulares del lenguaje sonoro (citados en Huwiler, 2016, p. 99). 


\section{DISERTACIONES}

ESTUDIOS

El papel del sonido en la comunicación: contribución, funciones y efectos

ISSN: $1856-9536$

Doi: http://dx.doi.org/10.12804/revistas.urosario.edu.co/disertaciones/a.6407

Volumen 12, Número 2 / Julio-diciembre 2019

Versión PDF para imprimir desde

http://revistas.urosario.edu.co/index.php/disertaciones

Bajo este argumento, nuestro análisis se decantó por la perspectiva audionarratológica, pues esta permite explorar la estructura narrativa del lenguaje sonoro, así como el potencial de sus elementos para desempeñar distintas funciones narrativas. De esta manera se puede observar cómo sonido e imagen se conectan para crear sentido.

De ahí, un análisis integral del lenguaje sonoro cinematográfico debe considerar la totalidad de sus elementos porque, como señala Tarkowsky, "[...] ningún elemento cinematográfico puede tener significado si lo tomamos aisladamente: es la película tomada en su conjunto lo que es una obra de arte, y si podemos hablar de los elementos que la componen, lo hacemos un tanto arbitrariamente, separándolos artificialmente para poder discutirlos a nivel teórico" (2009, p. 114).

En consecuencia, este análisis parte de los sistemas de signos que integran el lenguaje sonoro y concretamente se detiene en el sistema de signos de la voz.

Chion (2004) destaca la importancia narrativa de la voz en tanto su sonido atrapa la atención del espectador "aislándola y estructurando en torno a ella la percepción del todo: trata de pelar el sonido para extraer el significado, intenta siempre localizar y, si es posible, identificar la voz" (p. 18).

En este sentido, Chion elabora el concepto de vococentrismo, referido al rango privilegiado de la voz humana sobre los demás sonidos. "La voz humana jerarquiza todo a su alrededor". En términos del autor, el vococentrismo es "la escucha humana y, necesariamente, el propio cine hablado en su inmensa mayoría” (2004, pp. 18-19).

El sonido de la voz en el cine se potencia por medio de una manipulación electroacústica, esta refuerza su presencia en relación con los demás elementos sonoros. No se trata solamente de hacer la voz inteligible, sino de destacarla sobre los demás sonidos.

La voz por encima de los demás elementos sonoros en una película automáticamente coloca al resto de los sonidos en un segundo plano de atención. En el aspecto narrativo, el sonido de la voz manda, pues esas voces "gritan, susurran, lloran, acarician, amenazan, imploran, seducen, ordenan, ruegan, rezan, hipnotizan, confiesan, aterrorizan, declaran..." (Dolar, 2007, p. 32).

\section{Pensar la escucha de la voz}

El filósofo alemán Rudolph Arnheim teorizó acerca del lenguaje sonoro y lo describió como “[...] un mundo seductor y excitante que está en posesión no sólo del mayor estímulo que conoce el hombre para los sentidos, la música, la armonía y el ritmo, sino que, al mismo tiempo, es capaz de dar una descripción de la realidad por medio de ruidos y con el más amplio y abstracto medio de divulgación de que es dueño el hombre: la palabra” (Arnheim, 1980, p. 16).

Para Arnheim, la entonación y la impostación de las palabras, a través de su vehículo: la voz, llegan a considerarse como formas de efectos sonoros. En este juego de seducción del auditorio, el espacio lúdico de lo sonoro se despliega para cambiar la voz, simular y representar otras voces. La oralidad discurre en una suerte de performance: donde el tono de la palabra, por ser más elemental, resulta más importante que su significado. "Sobre la gente sencilla, influye más la expresión de la voz de un orador que el contenido de su discurso" (Arnheim, 1980, p. 24).

El tono se percibe como una sensación acústica, provocada en el oído por el número de vibraciones por segundo de la onda sonora. Las variaciones entre los diversos tonos producen bajas frecuencias (tonos graves) y altas frecuencias (tonos agudos). Por ejemplo, un tono grave lo posee el trombón, mientras uno agudo el clarinete (Rodero, 2005, p. 24). 


\section{DISERTACIONES}

ESTUDIOS

El papel del sonido en la comunicación: contribución, funciones y efectos

ISSN: $1856-9536$

Doi: http://dx.doi.org/10.12804/revistas.urosario.edu.co/disertaciones/a.6407

Volumen 12, Número 2 / Julio-diciembre 2019

Versión PDF para imprimir desde

http://revistas.urosario.edu.co/index.php/disertaciones

Además del tono, los sonidos, entre ellos la voz humana, poseen otras cualidades subjetivas fundamentales para su expresividad: intensidad, duración y timbre.

Rodero (2005) define la intensidad del sonido como la sensación auditiva, en forma de energía, que depende de la propiedad sonora de la amplitud que genera la onda sonora.

Por su parte, la duración se refiere al tiempo de expansión que este requiere para ser percibido. Esta cualidad "[...] convierte en perceptible el sonido y nos proporciona una sensación de rapidez o lentitud" (Rodero, 2005, p. 23).

Por último, el timbre es el resultado de la combinación entre caracteres acústicos y sensaciones auditivas. Para Rodríguez Bravo, "la armonicidad es el distinto grado de limpieza y agradabilidad que percibimos al escuchar un sonido compuesto [...]”. Mientras más armónica es la gama de frecuencias, mayor es la sensación agradable y limpia que transmite una voz (Rodero, 2005, p. 26).

De las cuatro cualidades subjetivas de la voz, tono y timbre son las que mejor se perciben y aportan mayor significación. "[...] el timbre nos remite rápidamente a la realidad que representa un sonido. Por ejemplo, una voz se diferencia claramente de otra por su timbre y tono, no por su intensidad o duración. Conocemos a alguien que nos llama por teléfono por su timbre y tono, no porque hable más alto o más rápido. Por eso, cuando calificamos la voz de alguien siempre aludimos al timbre: ¡Qué voz más ronca! O al tono: ¡Qué voz más grave!” (Rodero, 2005, p. 32).

\section{La voz de Dios}

Mladen Dolar (2007) habla acerca de la voz del padre, esa voz que ordena y crea obligaciones. El autor la relaciona con la voz de Dios y al mismo tiempo la equipara con el sonido del 'shofar'.

Si ha de haber una ley fundante, un pacto, la voz tiene que desempeñar en ella un papel crucial. Este es el problema que plantea Lacan en su seminario sobre la angustia inspirándose en el impactante análisis que Theodor Reik ha hecho del shofar, un cuerno primitivo empleado en los rituales religiosos judíos y uno de los instrumentos de viento más antiguos (2004, p. 281).

Es preciso entonces reconocer, en el sonido del shofar, la voz del padre, el grito de agonía del padre primitivo, de la horda primitiva. El que retorna para hacerse presente y a la vez para sellar la fundación de su ley. La ley misma, en su forma pura, antes de ordenar nada específico, halla su epítome en la voz, la voz exige obediencia absoluta, aunque sea insensata en sí (Dolar, 2007, pp. 67-68).

La Biblia menciona el sonido del shofar en numerosas ocasiones. Cada una de ellas es notable y tiene lugar en momentos dramáticos. Unos de los más significativos es la fundación de la Ley. Cuando Moisés recibe las Tablas de la Ley en el monte Sinaí, el sonido del shofar dio testimonio de la presencia de Dios ante un pueblo que no pudo oír sino ese tremendo sonido. El shofar fue la voz en medio del trueno:

Al tercer día, al rayar el alba, hubo truenos y relámpagos y una densa nube sobre el monte y un poderoso resonar de trompeta; y todo el pueblo que estaba en el campamento se echó a temblar (Éxodo 19:16).

Todo el pueblo percibió los truenos y relámpagos, el sonido de la trompeta y el monte humeante, y tem-

blando de miedo se mantuvo a distancia y le dijo a Moisés: "Háblanos tú, y escucharemos. Pero no dejes que nos hable Dios, o moriremos" (Éxodo 20:18).

De modo que el shofar, cuyo sonido es más fuerte que todos los truenos, está ahí como la voz sin contenido que se adhiere a la Ley, soporte de la ley, que sostiene su letra. Hay, en este momento inaugural, una división entre 


\section{DISERTACIONES}

ESTUDIOS

El papel del sonido en la comunicación: contribución, funciones y efectos

ISSN: $1856-9536$

Doi: http://dx.doi.org/10.12804/revistas.urosario.edu.co/disertaciones/a.6407

Volumen 12, Número 2 / Julio-diciembre 2019

Versión PDF para imprimir desde

http://revistas.urosario.edu.co/index.php/disertaciones

la voz que oye el pueblo, como presencia terrible y dominante, y la Ley a la que solo Moisés puede 'dar sentido'. Pero no hay ley sin voz (Dolar, 2007, p. 69).

Por lo tanto, la representación de la voz de Dios en el cine, de género épico, bíblico y mitológico, necesariamente ha tenido en cuenta el sonido del shofar, cuya traducción convencional es trompeta.

\section{La voz de Dios en el cine de Hollywood}

En un número considerable de películas, aparece la voz del que todo lo ve sin estar. Dentro del cine clásico destaca The Ten Commandments (Cecil B. DeMille, 1956). Charlton Heston, además de interpretar a Moisés, es la voz poderosa y amenazante de Jehová. Heston da voz a Dios y recrea ese pasaje fundacional descrito en Éxodo 24:16.

Y la gloria del sEÑon reposó sobre el monte Sinaí, y la nube lo cubrió por seis días; y al séptimo día, [Jehová]

llamó a Moisés de en medio de la nube.

Y al entrar Moisés en la tienda de reunión para hablar con el Señor, oyó la voz que le hablaba desde encima

del propiciatorio [que estaba] sobre el arca del testimonio, de entre los dos querubines, [y] Él le habló.

Otra cinta para destacar es The Bible in The Beginning (John Huston, 1966), una de las películas más ambiciosas del cine clásico de Hollywood, donde la voz de Dios es protagonista. Su productor, Dino De Laurentiis, se dio a la tarea de llevar a la pantalla una parte del 'Libro de libros', solo la mitad del Génesis. Para ello contó con un reparto destacado: Ava Gardner, Peter O’Toole, George C. Scott.

Houston, además de dirigir el filme, interpreta la voz de Dios, del narrador y representa el papel de Noé. La impactante voz de Dios está presente en la mayoría de los pasajes de esta cinta: la expulsión del hombre del paraíso terrenal, la expansión del pueblo de Dios por la faz de la Tierra con el sacrificio de Abraham, el diluvio universal o la destrucción de Sodoma y Gomorra se revelan en este filme.

John Huston confió en poseer el grano de la voz, es decir, en tener esa voz con "una doble postura, una doble producción: como lengua y como música” (Barthes, 2009, p. 301). El 'grano' sería, entonces, la materialidad del cuerpo hablante, por encima de lo inteligible, de lo expresivo que arrastra directamente lo simbólico. Aunque Barthes se refirió con este término a la voz de un cantante, consideramos que Houston posee la sonoridad y la dicción para representar la voz de Dios, pues tiene el balance (expresividad, dramatismo, personalidad y subjetividad) propicio para el goce de quienes lo escuchamos como voz de Dios.

Las dos películas se caracterizan porque el personaje-voz de Dios es el acusmaser. La magia y el poder del acusmaser provienen de que no lo vemos, pero lo oímos en todas las direcciones. "De todos los sentidos, el oído es, probablemente, el que más pronto se despierta, pues funciona ya en el estado fetal, en el que el ser humano capta la voz de la madre, que reconocerá después de haber nacido" (Chion, 2004, p. 29).

Pierre Schaeffer (2003) empleó el término acusmática en su Tratado de los objetos musicales. En este define los sonidos acusmáticos como aquellos sonidos cuya fuente emisora no es visible.

En sentido antiguo, la palabra acusmático se refiere al nombre dado a una secta pitagórica cuyos adeptos escuchaban a su maestro hablar detrás de un cortinaje. Sin embargo, la regla del prohibido ver existe en muchos ritos y religiones y convierte al maestro, al dios o al espíritu en una voz acusmática. De esta manera, un dios que todo lo ve y todo lo sabe es un acusmaser (Chion, 2004, pp. 30-38). 


\section{DISERTACIONES}

ESTUDIOS

El papel del sonido en la comunicación: contribución, funciones y efectos

ISSN: $1856-9536$

Doi: http://dx.doi.org/10.12804/revistas.urosario.edu.co/disertaciones/a.6407

Volumen 12, Número 2 / Julio-diciembre 2019

Versión PDF para imprimir desde

http://revistas.urosario.edu.co/index.php/disertaciones

La idea de un Dios omnipresente y omnisciente de las religiones judaica, cristiana e islámica se basa en el poder otorgado por la falta de cuerpo físico, pero ¿cómo puede una voz concentrar tanto poder? Tal vez porque esa voz sin fuente perceptible, la del acusmaser, nos traslada a una fase arcaica, original, de los primeros meses de vida e incluso de antes de haber nacido, donde la voz lo es todo y se encuentra por doquier.

No obstante, en algunas películas la voz de Dios tiene cuerpo. A esto Chion le denomina desacusmatización. En una especie de acto simbólico, la encarnación de la voz le confiere el destino de los mortales, pues "le asigna un lugar y le dice: este es tu cuerpo, morarás en él y en ninguna otra parte” (Chion, 2004, pp. 39-41). Dios toma un cuerpo, y con ello hace una concesión a los mortales para ser más cercano, más democrático.

Un ejemplo del Dios del siglo xxı es Morgan Freeman, como lo demuestra el análisis de las cintas Bruce Almighty (2003) y Evan Almighty (2007). Freeman también es el protagonista de la serie documental de National Geographic Channel The Story of God (2016). En la serie explora la fe, las creencias y el poder de dios en varias culturas y religiones. Los títulos de algunos episodios son de por sí sugerentes: "Beyond Death”, “Apocalypse”, "Who is God?", "Creation”, "Why Does Evil Exist?", "The Chosen One”, "Heaven and Hell” y "Proof of God".

No es casual, dada su presencia y versatilidad actoral, que en su prolífica filmografía Morgan Freeman haya interpretado roles de personajes maduros, sabios y que inspiran confianza. Pero, como ya se ha mencionado, no todo es imagen, su voz ha hecho que lo admiremos también en su carácter de acusmaser. Tanto es así que su voz se ha colado en la rutina diaria a través de una app, del asistente virtual de navegación vehicular Waze. Con ello, ha hecho realidad la protectora frase de nuestras madres y abuelos cuando nos dicen: “iVe con Dios!”. Él guía el camino para llegar a nuestro destino.

Voces como la de Freeman llaman la atención e inspiran confianza, pero ¿a qué se debe la fascinación que provocan? La explicación se encuentra en las investigaciones de Klofstad, Anderson y Nowicki (2015) "Influence of Candidate Age on Vote Choice" e "Influence of Perceived Strength, Competence, and Age on Preference for Candidates with Lower Voices".

En este estudio, Klofstad y sus compañeros grabaron a hombres y mujeres enunciando: "Te pido que votes por mí este mes de noviembre". Posteriormente, cuando las grabaciones fueron escuchadas por los individuos que conformaron la muestra, todos se decantaban por las versiones más profundas de la frase con independencia del género. Y es que la profundidad de la voz se asocia a cualidades positivas como la integridad, la competencia y el poder físico (Klofstad, et al., 2015).

En los resultados, publicados en Perceptions of Competence, Strength, and Age Influence Voters to Select Leaders with Lower-Pitched Voices, los investigadores concluyen que los votantes prefieren líderes con voces de tono bajo o grave, porque se les percibe como de carácter fuerte, con mayor fortaleza física, más competentes y con mayor integridad. Además, plantean una hipótesis alternativa, por comprobarse en otro estudio, que los candidatos con voces de tono bajo o grave se perciben con mayores conocimientos y, por lo tanto, más sabios y experimentados. Dadas estas conclusiones, no es de extrañar que Morgan Freeman encarne la voz de Dios, su voz "se asocia a una figura dominante y viril", argumenta Klofstad, Anderson y Nowicku (2015).

En México, Rubén Mayo -locutor, actor y director de doblaje- es la voz en español de Freeman, tanto de sus películas como de la serie documental The Story of God (2016). En España este papel lo realizó el actor de doblaje Pepe Mediavilla (1940-2018), que entre muchas otras voces también dio voz a Gandalf. Las voces de Mayo y Mediavilla 


\section{DISERTACIONES}

ESTUDIOS

El papel del sonido en la comunicación: contribución, funciones y efectos

ISSN: $1856-9536$

Doi: http://dx.doi.org/10.12804/revistas.urosario.edu.co/disertaciones/a.6407

Volumen 12, Número 2 / Julio-diciembre 2019

Versión PDF para imprimir desde

http://revistas.urosario.edu.co/index.php/disertaciones

concuerdan con el perfil del estudio citado, son voces poderosas, de tono bajo o grave, enérgicas, con autoridad y dignas de infundir respeto; sin sonar déspotas.

\section{Propuesta de modelo teórico-metodológico para el análisis de la voz en el cine}

Desde una perspectiva semiótica, el análisis del lenguaje cinematográfico consiste en el examen de los diversos componentes de una película: imagen, sonido, montaje, puesta en escena, narración. Dicho análisis puede basarse en un solo elemento, en varios de ellos, por separado o de manera simultánea (Zavala, 2010, p. 66).

Para el enfoque posnarratológico, derivado en parte de la semiótica, "las narrativas no son meramente una forma literaria o un medio de expresión, sino un modo fenomenológico y cognitivo de conocimiento de sí mismo y del mundo" (Nünning \& Nünning, citados en Huwiler, 2005, p. 51).

En esta línea, el análisis narratológico es aplicable a múltiples objetos de estudio, desde la observación de las historias que cuenta y escucha la gente en determinadas formas y contextos, los relatos cotidianos, testimonios, conversaciones, hasta cuentos de hadas, novelas, cuentos cortos, biografías, libros de historia, cómics y películas (Nünning \& Nünning, citados en Huwiler, 2005, p. 51).

Por ello, el abordaje interdisciplinario en el estudio de la narrativa abre camino a nuevas preguntas sobre la estructura narrativa, su construcción verbal, visual y su relación con los contextos en los que se produce e interpreta (Herman, citado en Huwiler, 2005, p. 51).

Recientemente, teóricos de la narratología posclásica han aportado nuevos conceptos, técnicas y categorías de análisis a este paradigma. Los investigadores Mildorf y Kinzell (2016a) abonan a este campo de estudios la formación de una nueva disciplina basada en lo sonoro, la audionarratología.

La audionarratología explora la relación entre la narrativa y las formas de expresión oral y auditiva de los media, sus distintos géneros y las estructuras narrativas, así como las funciones que desempeñan. En este sentido, proponen dar un "giro acústico" para abordar dichos objetos de estudio.

El foco de interés son los medios auditivos y los canales orales/auditivos. Para ello, la audionarratología desplaza las preguntas clásicas de los estudios culturales y literarios sobre las relaciones entre texto-imagen y lo visual, en términos de la intertextualidad y "se diferencia [...] por centrarse en la relación entre las formas y funciones del sonido como narrativa” (Mildorf \& Kinzel, 2016a, p. 8).

Es así que la audionarratología es entendida como un 'término paraguas' para aquellos estudios que toman en cuenta las formas y funciones del sonido desde un enfoque narrativo. El sonido en este contexto incorpora todo el espectro del sonido estructurado, desde la música o el lenguaje hablado, las características prosódicas de las voces, pasando por los sonidos que emanan de fuentes reconocibles o el ruido más o menos indeterminado, hasta la manipulación electroacústica de todos ellos (Mildorf \& Kinzel, 2016a, p. 8).

Mediante sus conceptos y métodos destaca la importancia del sonido en los textos ficcionales, así como las cualidades sonoras y las características, percibidas o imaginadas, por el auditorio de las voces de narradores y personajes.

Entre sus áreas de interés se encuentran: el cine, los dramas televisivos, videos y juegos de computadora; géneros musicales dramáticos como musicales y ópera; los audiolibros, los podcasts, la lectura de textos poéticos 


\section{DISERTACIONES}

ESTUDIOS

El papel del sonido en la comunicación: contribución, funciones y efectos

ISSN: $1856-9536$

Doi: http://dx.doi.org/10.12804/revistas.urosario.edu.co/disertaciones/a.6407

Volumen 12, Número 2 / Julio-diciembre 2019

Versión PDF para imprimir desde

http://revistas.urosario.edu.co/index.php/disertaciones

y literarios; géneros de música popular, performances e instalaciones sonoras; narrativas digitales en internet, audioguías de museos y viajes.

Mildorf y Kinzel proponen tres ejes o trayectorias entre los que opera esta disciplina (2016a, p. 9):

1. De medios audiovisuales como la televisión, el cine o la computación a medios puramente auditivos como la radio.

2. De obras de corte literario/artístico como la ópera, canciones populares, radiodramas o poesía a los llamados géneros pragmáticos como las audioguías.

3. De formas verbales a no verbales de expresión, como serían los audiolibros en el primer caso y las instalaciones sonoras en el segundo.

Este estudio se sitúa en el primero de estos ejes, sin embargo, independientemente del medio o género, el interés de la disciplina abarca todas las manifestaciones narrativas en, y a través de, los medios y géneros.

En este sentido, los signos y señales acústicas aportan significado al mundo ficcional creado y le confieren verosimilitud. Por ello, las voces de los personajes, la música, el silencio y los efectos o ruidos empleados para ambientar o crear atmósferas son elementos, que, junto a la imagen, "contribuyen a una experiencia simultánea y multimodal de la estructura narrativa" de la película y de sus diferentes niveles narrativos, tanto dentro de la diégesis como fuera de ella (Mildorf \& Kinzel, 2016b, p. 312).

\section{Descripción de la muestra}

En principio se consideró una línea temporal de 1936 a 2016, de este lapso se eligieron 22 películas donde figura la voz de Dios, o este aparece como personaje. El siguiente paso consistió en extraer los fragmentos donde se representa al personaje-voz de Dios.

Para la selección de la muestra, se tomaron en cuenta los siguientes criterios:

1. Abarcar distintos géneros cinematográficos.

2. Dar un panorama amplio de las distintas voces con las que se ha caracterizado al Dios occidental en las cintas hollywoodenses.

3. Contar con características donde, además de la voz, se emplearan otros elementos del lenguaje sonoro para caracterizar al personaje.

Bajo estos criterios, la muestra definitiva quedó integrada por doce filmes de los que a continuación se hace una breve descripción.

1. The Green Pastures (Marc Connelly \& William Keighley, 1936). The Green Pastures, conocida en castellano como Los verdes prados, presenta distintos pasajes del Antiguo Testamento, entre ellos, el arca de Noé y la creación. Del drama, escrito por Marc Sheridan Gibney y Marc Connelly desde la perspectiva rural de los negros americanos, se retoma una escena de 1'46" con la conversación entre Noé y el Señor. Dios es representado por el artista afroamericano Rex Ingram. La música es del compositor y director de orquesta de origen checo Erich Wolfgang Korngold.

2. The Ten Commandments (Cecil B. DeMille, 1956). En este drama la voz de Dios es representada por Charlton Heston, quien a su vez interpreta el papel de Moisés. El reconocido compositor Elmer Bernstein se encargó de la música. Para el análisis se eligió un fragmento de 3'01" donde Moisés recibe las Tablas de la Ley de 


\section{DISERTACIONES}

ESTUDIOS

El papel del sonido en la comunicación: contribución, funciones y efectos

ISSN: $1856-9536$

Doi: http://dx.doi.org/10.12804/revistas.urosario.edu.co/disertaciones/a.6407

Volumen 12, Número 2 / Julio-diciembre 2019

Versión PDF para imprimir desde

http://revistas.urosario.edu.co/index.php/disertaciones

Dios. La divinidad se hace presente con música, viento y truenos. Años después Charlton Heston volvió a interpretar a Dios en Almost an Angel de John Cornell (1990).

3. Oh, God! (Carl Reiner, 1977). Comedia ligera basada en la novela homónima de Avery Corman, con guion escrito por Larry Gelbart y con música del compositor e intérprete Jack Elliott. Un Dios simpático y benevolente, interpretado por el veterano actor George Burns, se presenta al gerente de una tienda para pedirle que esparza su mensaje en el mundo moderno. Para el análisis se eligió una secuencia donde los personajes conversan mientras viajan en automóvil (1'20"). La versión hispana se dio a conocer bajo el título ¡Dios Mío! A esta primera siguieron dos secuelas: Oh, God! Book Two (Gilbert Cates, 1980) y Oh, God! You Devil (Paul Bogart, 1984).

4. Night Train to Terror (Jay Schlossberg-Cohen, 1985). Dentro del género de terror fantástico un vagón de tren sirve de escenario a dos pasajeros peculiares, Dios y Satanás, para exponer sus argumentos sobre las opciones que presentan a los seres humanos. Durante el trayecto con trágico destino, Dios -interpretado por Ferdy Mayne- y Satanás revisan tres casos para decidir si sus protagonistas merecen el cielo o el infierno. Eddy Manson se encargó de la música de la cinta. De esta se extrajo un fragmento de 1'28” de los cuatro segmentos donde intervienen los personajes mencionados.

5. Switch (Blake Edwards, 1991). Comedia fantástica, escrita y dirigida por Blake Edwards. Después de ser asesinado por tres de sus examantes, Steve Brooks, un mujeriego chauvinista, es regresado a la Tierra para redimirse y encontrar una mujer que lo ame de verdad; de no conseguirlo su alma será enviada al infierno. La escena analizada tiene lugar en un purgatorio oscuro, donde la voz de Dios emana de una columna de luz brillante (1'22"). La música es del afamado Henry Mancini. La cinta presenta un giro innovador al representar a Dios alternando una voz masculina, Richard Provost, con una femenina, Linda Gary.

6. The Prince of Egypt (Brenda Chapman, Steve Hickner, Simon Wells, 1998). Drama musical animado que retrata la vida de Moisés, desde su nacimiento hasta el descubrimiento de su origen hebreo. De esta cinta se analiza un fragmento (4' 10") que corresponde a una pasaje del Éxodo. Este narra el encuentro de Moisés con Dios, quien le habla a través de la zarza ardiente en el monte sagrado Horeb, también conocido como monte Sinaí. La música de la película es de Hans Zimmer, considerado uno de los talentos más innovadores de Hollywood.

7. Dogma (Kevin Smith, 1999). En esta comedia negra un hombre común tiene la misión de salvar a la humanidad de ser aniquilada por dos ángeles renegados. En medio del caos y la destrucción total aparece Alanis Morissette en el papel de la divinidad, un-a Dios-a que no necesita de palabras para comunicarse. El fragmento analizado dura 7'. La música de la película es del compositor Howard Shore.

8. Bruce Almighty (Tom Shadyack, 2003). Bajo el título en castellano: Como Dios (España) o Todopoderoso (Hispanoamérica). En esta comedia el papel de Dios es interpretado por Morgan Freeman. Un periodista insatisfecho reniega y ridiculiza a Dios luego de un mal día; entonces se le presenta la figura humanizada de Dios que lo invita a tomar su lugar y le transfiere sus poderes. La música es del compositor y director de orquesta John Debney. Para el análisis fue seleccionada una secuencia de 2' 27", en esta el protagonista arrepentido de ser Dios se rinde ante la voluntad divina. La secuela de esta cinta Evan Almighty se estrenó en 2007, de nuevo Freeman interpretó el rol de Dios. 


\section{DISERTACIONES}

ESTUDIOS

El papel del sonido en la comunicación: contribución, funciones y efectos

ISSN: $1856-9536$

Doi: http://dx.doi.org/10.12804/revistas.urosario.edu.co/disertaciones/a.6407

Volumen 12, Número 2 / Julio-diciembre 2019

Versión PDF para imprimir desde

http://revistas.urosario.edu.co/index.php/disertaciones

9. A Little Bit of Heaven (Nicole Kasell \& Gren Wells, 2011). En esta comedia romántica, una joven con cáncer terminal se enamora del médico que la trata. La anestesia la lleva por unos momentos al cielo donde conversa con un dios encarnado en Whoopi Goldberg. La escena analizada tiene una duración de 2' 07'. Más adelante en el film vuelve a conversar con Ella, luego de casi ser arrollada por un vehículo. El brasilero Heitor Pereira fue el compositor de la música. Goldberg había interpretado a Dios en la película $A$ Very Merry Muppet's Christmas Movie (2002).

10. Exodus: Gods and Kings (Ridley Scott, 2014). Película de acción que relata el levantamiento de Moisés en contra del faraón egipcio y la jornada épica que emprende para liberar al pueblo judío. La historia fue recreada a partir del Éxodo. De esta cinta se retoma el momento en que Moisés se encuentra a Malak en el monte sagrado. Durante la conversación Moisés se da cuenta que Dios habla a través del niño (2’ 01”). Malak (ángel o mensajero en hebreo) fue interpretado por Isaac Andrews. La música fue del compositor vasco Alberto Iglesias.

11. Noah (Darren Aronofsky, 2014). Protagonizada por Russell Crowe con música de Clint Mansell cuenta el conocido pasaje bíblico. En sueños Noé recibe mensajes divinos que le advierten de la destrucción de la vida sobre la Tierra, del Diluvio y de su misión: construir el arca y salvar a los inocentes. En distintos momentos del filme, Dios se comunica a través de sueños, de efectos sonoros y visuales, nunca mediante una manifestación verbal.

12. Army of One (Larry Charles, 2016). Comedia con tintes biográficos basada en la historia de Gary Faulkner, un ciudadano estadounidense con la misión divina de acabar con Osama bin Laden. De esta cinta se analiza la escena (2' 26") en que Dios, interpretado por Russell Brand, lo insta a hacer justicia por propia mano.

\section{Variables de análisis}

En los fragmentos de las películas se analizaron las siguientes variables:

Voz. Este sistema de signos se considera de forma independiente al sistema del lenguaje, pues sus cualidades sonoras generan significado por sí mismas. A las ya mencionadas se suman el idiolecto (expresión de la forma de ser, de pensar, de gustos y necesidades), la pronunciación (acentos y/o dialectos) y la entonación (forma de enfatizar palabras y melodía del fraseo).

En relación con la voz de un personaje, la combinación de estas cualidades genera en el espectador una experiencia sensorial que juega un papel relevante en la construcción de significados (Mildorf \& Kinzel, 2016b, pp. 314-315).

Música. Rodero y Soengas (2010) describen cuatro funciones principales de la música: funcional (al respaldar y potenciar acciones), expresiva (cuando se emplea para crear y reforzar emociones), descriptiva (recrea la realidad) y narrativa (estructura el relato). La música, además de denotar ambientes y connotar atmósfera, puede desempeñar distintas funciones narrativas para crear significados.

Punto de escucha. Su función principal es localizar la acción, fijarla para dar al oyente la misma perspectiva. Determinar el punto de escucha es una decisión fundamental en el montaje, pues establece las relaciones espaciales a partir de la proxémica y kinésica. También jerarquiza los objetos dentro de la escena para darles diferentes grados de significación dentro del relato. En este sentido, la distribución espacial de las señales puede significar 


\section{DISERTACIONES}

ESTUDIOS

El papel del sonido en la comunicación: contribución, funciones y efectos

ISSN: $1856-9536$

Doi: http://dx.doi.org/10.12804/revistas.urosario.edu.co/disertaciones/a.6407

Volumen 12, Número 2 / Julio-diciembre 2019

Versión PDF para imprimir desde

http://revistas.urosario.edu.co/index.php/disertaciones

más que la ubicación espacial de los objetos o personas en un entorno realista y adquirir funciones narrativas (Rodero \& Soengas, 2010, p. 201).

Mediante el punto de escucha se distingue la escena principal de la secundaria (main frame y outer frame). Este puede ser fijo o móvil. Cuando es móvil puede presentarse en tres variantes: genera en el oyente la sensación de moverse horizontal o verticalmente en un espacio; cuando da un salto brusco de un lugar a otro en el mismo espacio sonoro; o cuando brinca de un espacio a otro completamente diferente (Rodríguez Bravo, citado en Rodero \& Soengas, 2010, p. 203).

Efectos sonoros y ruidos. Además de los ruidos y sonidos que provienen de su fuente original, este sistema de signos abarca a aquellos generados electroacústicamente en tanto estos hacen referencia a sonidos reales.

Recursos técnicos. La manipulación electroacústica solo se considera como sistema de signos cuando es posible detectarla y atribuirle un significado narrativo. Su función principal es matizar y enriquecer la expresividad del sonido, apoyando la caracterización de espacios y personajes por medio de la manipulación o distorsión de un sonido. Gracias a ellos se puede transformar por completo el significado de un sonido determinado.

Silencio. El silencio también tiene un gran potencial narrativo y llega a tener efectividad si es manejado adecuadamente.

\section{Una voz, distintas manifestaciones: palabra, efecto, música y silencio}

Entre los hallazgos destaca el uso de recursos sonoros, más que visuales, para introducir a Dios en la diégesis de la historia. El personaje-voz de Dios se expresa a través de la voz, los efectos de sonido, la música y el silencio, explotando los sistemas de signos del lenguaje sonoro.

En relación con los resultados por cada variable analizada, se observa lo siguiente:

Voz. Cuatro películas, de las doce películas analizadas, presentan la voz de Dios acusmatizada, sin cuerpo. En este sentido, el personaje-voz Dios es un acusmaser (Chion, 2004, pp. 29-30). En The Ten Commandments la voz grave, pausada y poderosa de Heston es un ejemplo de esta caracterización de Dios en el cine clásico de Hollywood. El fraseo del actor está caracterizado por un ritmo de acentos muy marcados asociados a energía y misticismo.

La originalidad del acusmaser de Switch radica en la representación de una divinidad con doble naturaleza: femenina/masculina. La alternancia de las voces se acentúa por la combinación de tonos graves y agudos, empleados para provocar la sensación de altura espiritual.

Por su parte, en The Prince of Egypt se manifiesta en un principio como una voz suave y paternal para luego transformarse en colérica. El enfado se manifiesta acelerando la velocidad e intensidad del fraseo. Ante el temor evidente de Moisés, la voz se dulcifica, entonces la voz se ralentiza y suaviza la intensidad.

Por último, el acusmaser en Noah tiene como rasgo distintivo la nula manifestación vocal; el personaje-voz de Dios se expresa mediante efectos de sonido y ruidos propios de la naturaleza.

Las siete restantes desacusmatizan a Dios al encarnarla en la presencia física de un actor.

La primera corresponde a una etapa temprana del cine hablado, en The Green Pastures (1936) se representa a un Dios con características antropomórficas que lo sitúan al mismo nivel del hombre, por lo menos en apariencia física. La diferencia se expresa por una voz enfática que enuncia: ¡Así es como debe ser! Su autoridad está remarcada por el timbre, el tono grave y la intensidad. 


\section{DISERTACIONES}

ESTUDIOS

El papel del sonido en la comunicación: contribución, funciones y efectos

ISSN: $1856-9536$

Doi: http://dx.doi.org/10.12804/revistas.urosario.edu.co/disertaciones/a.6407

Volumen 12, Número 2 / Julio-diciembre 2019

Versión PDF para imprimir desde

http://revistas.urosario.edu.co/index.php/disertaciones

Un giro radical se observa en la cinta Oh, God! La voz desacusmatizada en el veterano actor George Burns se caracteriza por un tono grave y un timbre rico en armónicos que sugieren una juguetona vitalidad y confianza. En cambio, en Night Train To Terror, también se presenta a un Dios de edad avanzada pero serio y firme, con una voz grave, su ritmo de fraseo es pausado y de baja intensidad cuando se refiere a cuestiones trascendentes.

En el fragmento analizado de Bruce Almighty, el protagonista asciende al cielo y se encuentra con un dios desacusmatizado en Morgan Freeman. Su tono grave sugiere profundidad, energía y denota autoridad. Su fraseo con un ritmo e intensidad regular agrada al oído e inspira confianza. Su voz lo muestra como un Dios amable, comprensivo, bromista y empático.

De nuevo una voz grave y ronca, ahora femenina, representa a la divinidad. Whoopi Goldberg personifica a una divinidad bonachona, agradable e incluso maternal en A Little Bit of Heaven.

La voz de Russell Brand se distingue, de entre las demás cintas, por fraseo acelerado con una entonación media que sube a tonos agudos en varios momentos de Army of One.

La cinta Dogma rompe con los esquemas para representar al personaje-voz de una divinidad, sin género, que toma un cuerpo femenino para manifestarse. Su rasgo innovador consiste en expresarse mediante recursos vocales, sin emplear la palabra para comunicarse.

Una excepción la encontramos en Exodus (2014), la voz de Dios (acusmatizada) se manifiesta a través de la voz de un niño, y en este sentido podría considerarse desacusmatizada. Por ello, consideramos que responde a una doble representación porque el timbre se mantiene y las variaciones se observan en el tono, intensidad y duración.

Música. La dimensión emocional de la música se usa para generar empatía en el espectador e inspirar diversas emociones: temor, tranquilidad, confianza. También para enfatizar la voz de Dios y para provocar el aumento progresivo de la tensión dramática. En The Ten Commandments, la música se emplea para reforzar la acción, a cada mandamiento antecede una secuencia musical que combina instrumentos de cuerdas, viento y percusiones; entre los instrumentos se distingue el corno, símbolo de la voz de la divinidad: el shofar, cuyo sonido es más fuerte que todos los truenos (Dolar, 2007, pp. 67-68).

En Switch, una música suave, tenue, de tonos agudos, sugiere una entrada etérea de la voz de Dios a escena. De forma similar, una música suave que inspira confianza, esta vez con un coro angelical, introduce la voz de Dios en The Prince of Egypt. Por el contrario, en Exodus y Night Train To Terror sirve para acentuar la atmósfera de suspenso y fatalidad. Estas cintas ejemplifican la función expresiva de este recurso para provocar emociones en el espectador.

Una transición musical se usa en términos narrativos para estructurar el relato y saltar de un mundo ficcional a otro en Bruce Almighty y en A Little Bit of Heaven. Los personajes pasan de la tierra al cielo y, de esta manera, la música también funciona para connotar sus procesos de transformación.

Por su parte, en las secuencias analizadas de Dogma y Noah, la música combina su función expresiva con la narrativa. En las dos cintas se utiliza la música para introducir a Dios a escena.

Finalmente, en Army of One la música funciona para ambientar el bar donde se aparece Dios, con una pieza country.

La ausencia de este recurso sonoro se observa solo en The Green Pastures y Oh, God!

Punto de escucha. The Green Pastures ejemplifica la forma en la que el punto de escucha jerarquiza las voces de los personajes dentro de la escena. Constata la noción del vococentrismo en el cine (Chion, 2004). Sin embargo, el sonido de un trueno poderoso sitúa la voluntad divina por encima de la humana. 


\section{DISERTACIONES}

ESTUDIOS

El papel del sonido en la comunicación: contribución, funciones y efectos

ISSN: 1856-9536

Doi: http://dx.doi.org/10.12804/revistas.urosario.edu.co/disertaciones/a.6407

Volumen 12, Número 2 / Julio-diciembre 2019

Versión PDF para imprimir desde

http://revistas.urosario.edu.co/index.php/disertaciones

En siete de los filmes analizados el punto de escucha se mantiene fijo en el diálogo, colocado en primer plano. The Prince of Egypt también es un caso de vococentrismo, pero en la cinta el punto de escucha comienza siendo móvil; una voz lejana y pausada se acerca a primerísimo primer plano.

En cambio, en The Ten Commandments el personaje-voz de Dios guía el punto de escucha móvil hasta ubicarse fuera de cuadro, como si la voz proviniera de las alturas.

En Dogma, el punto de escucha se mantiene en gran parte de la secuencia en la música con voces angelicales, aunque en los diálogos baja a segundo plano unos segundos, destaca el uso prolongado de la música en primero o primerísimo primer plano durante la secuencia.

Por último, en Noah el punto de escucha se sostiene en los efectos sonoros y ruidos que representan al personaje-voz. En ocasiones también se sitúa en la música en primer plano.

Efectos sonoros y ruidos. Los sonidos y ruidos son empleados para representar el personaje-voz de Dios, para caracterizarlo. Se prefiere asociar a la divinidad con sonidos de la naturaleza, los más recurrentes son el trueno, el fuego, el viento y el agua. También se observa su uso para desempeñar funciones narrativas, más que para describir objetos o ambientar una escena, por ejemplo, para introducir al personaje, o como elemento disruptivo para modificar la atmósfera de una escena, o un estado de ánimo. Por ejemplo, en The Green Pastures y en The Ten Commandments, donde la voluntad inapelable de Dios se impone con el sonido del trueno. En la segunda cinta, ráfagas de fuego graban los designios de Dios en las Tablas de la Ley que Moisés entregará a los judíos. El crepitar de la zarza ardiente indica la presencia de Dios en The Prince of Egypt. Otro efecto sonoro destacado proviene del bastón de Moisés. Este lo percute sobre el piso a la salida de la cueva y señala su convicción de cumplir la misión sagrada.

La lluvia es otro de los sonidos empleados para representar el poder condescendiente de la divinidad en Oh, God! Sin embargo, también expresa la ira divina en Noah, con el Diluvio.

En Night Train To Terror, se emplea el efecto de sonido del tren en marcha para ambientar la escena. También en Bruce Almighty encontramos este uso, en un desfibrilador, sin embargo, además de ser empleado en su carácter descriptivo, adquiere una función narrativa al ser el elemento de transición que regresa a Nolan a la tierra. En su aspecto funcional, el ruido producido por la palma de la mano indica a la protagonista de $A$ Little Bit of Heaven que tome asiento junto a Whoopi, la encarnación de la divinidad. En el mismo sentido, el Dios de Army of One apunta su dedo al televisor y lanza un rayo que lo apaga.

La originalidad de Dogma radica en el empleo de ruidos y sonidos que sustituyen al personaje-voz, algunos manipulados electroacústicamente, entre ellos destacan, un suspiro o aliento divino que restaura la vida en la Tierra, ruidos como un chasquido de lengua, el sonido de una leve sonrisa, el golpe de una palma contra la otra para cargar energía y regresar de la muerte a Bethany.

También en Noah se manifiesta la voz de Dios con efectos de sonido, un chorro de agua que brota de la tierra y hace crecer los árboles para construir el arca. Destaca una explosión y haz de luz, rayo que regresa al cielo a los seres luminosos convertidos en roca. Más adelante, el agua a borbotones también sustituye al personaje-voz, esta vez para destruir a la humanidad con el Diluvio. Por su parte, en Switch y Exodus no se emplea este recurso asociado a la divinidad, solo para describir algunos sonidos humanos, como los pasos sobre el piso mojado de Nolan, en la primera, y los balidos de ovejas y pasos de Moisés sobre la hierba en la segunda.

Recursos técnicos. Los más frecuentes son la reverberación y el eco. El uso de whoosh o ráfagas que enriquecen los atributos subjetivos del personaje-voz de Dios en The Ten Commandments, pues acentúan la solemnidad 


\section{DISERTACIONES}

ESTUDIOS

El papel del sonido en la comunicación: contribución, funciones y efectos

ISSN: $1856-9536$

Doi: http://dx.doi.org/10.12804/revistas.urosario.edu.co/disertaciones/a.6407

Volumen 12, Número 2 / Julio-diciembre 2019

Versión PDF para imprimir desde

http://revistas.urosario.edu.co/index.php/disertaciones

y profundidad de la inscripción de las Tablas de la Ley. En Switch, la sobreimposición de las voces es utilizada como un recurso para dar la idea de una divinidad sin género, la totalidad. La voz del Dios/Diosa es manipulada mediante la reverberación para realzar la infinitud de su presencia y la magnitud del espacio en que se encuentran: el purgatorio. En The Prince of Egypt, el eco y la reverberación en el interior de una cueva sugieren una atmósfera de profunda resonancia con el personaje-voz de Dios como un sonido envolvente que llega a Moisés desde todos los rincones, símbolo de su magnificencia. Destaca el efecto electroacústico empleado en sustitución de un rayo de luz, el sonido ilumina la cueva como si la voz de Dios entrara para abarcar la plenitud del espacio. Un efecto sonoro de ambiente sideral combinado con un viento suave, profundo y constante describe la escena en Bruce Almighty. En la misma cinta, un whoosh o ráfaga de viento se escucha mientras los paramédicos intentan regresar a Nolan del paro cardíaco.

El empleo de este sistema de signos para crear un sonido con un significado narrativo que apoya la caracterización del personaje lo encontramos en Dogma. Primero con el sonido de un grito que presenta al personaje dentro de la historia y, más adelante, con otro grito de la divinidad en un tono metálico, grave y poderoso con el que destruye al ángel rebelde. El sonido fue creado artificialmente para alejarse de cualquier similitud con un grito humano.

Un whoosh con sonido de campanas indica la entrada y salida de Dios a escena en Army of One.

Silencio. Esta variable solo se localiza en Dogma, donde la divinidad responde, en general, a las interpelaciones humanas con un profundo silencio. Realza su función narrativa dentro de la historia para caracterizar a un personaje que no se comunica con palabras.

\section{Consideraciones finales}

En el caso de las cintas analizadas, se observa el uso común de rasgos convencionales en la representación del personaje-voz de Dios a través de los años (1936-2016). Circunstancia, probablemente, atribuida al estereotipo marcado desde las instituciones religiosas, de un Dios masculino, de edad madura, enérgico, con autoridad absoluta, en ocasiones iracundo y punitivo.

La mayoría de las películas analizadas confirman que la voz, de acuerdo con el vococentrismo de Chion, ocupa el primer plano y a partir de ella se organizan los demás elementos sonoros; por lo tanto, es imprescindible estudiar sus cualidades como sistema de signos independiente del lenguaje en el análisis de un filme.

En este sentido, los resultados permiten concluir que las cualidades de la voz son un elemento decisivo para la credibilidad y comprensión del mensaje; como recurso sonoro, la voz da información al espectador sobre las características físicas y emocionales del personaje, así como de sus cualidades personales.

En general, se observan dos formas de representar la voz de Dios, en su carácter acusmático es una voz omnipotente y omnipresente; sin embargo, sobresale la tendencia a encarnar al personaje-voz de Dios otorgándole un cuerpo con características antropomórficas en el cual habitar. Si Dios nos hizo a su imagen y semejanza, el cine invierte esta figura y representa a Dios a nuestra imagen y semejanza. Por lo tanto, salvo algunas excepciones, es amable, cercano, comprensivo, con toques de simpatía y humor que infunden empatía.

Se prefieren voces de tonos bajos, de timbre ronco y ricas en armónicos de personas maduras para representar a la divinidad. Este dato constata la percepción de que las voces de tonos graves y timbre ronco son más creíbles y tienen más ascendencia sobre el espectador, pues se les adjudican atributos de autoridad, confianza y sabiduría. 


\section{DISERTACIONES}

ESTUDIOS

El papel del sonido en la comunicación: contribución, funciones y efectos

ISSN: $1856-9536$

Doi: http://dx.doi.org/10.12804/revistas.urosario.edu.co/disertaciones/a.6407

Volumen 12, Número 2 / Julio-diciembre 2019

Versión PDF para imprimir desde

http://revistas.urosario.edu.co/index.php/disertaciones

En relación con la música, en la mayoría de los filmes se trata de música extradiegética: usada para apoyar la acción o inducirla; para potenciar e incidir en las emociones del espectador; y para estructurar la historia (como elemento de transición entre escenas, estados de ánimo o para introducir al personaje o una acción, inscribir en piedra cada mandamiento, por ejemplo). En su función narrativa llama la atención el prolijo uso de la música para representar el personaje-voz de Dios.

Referente al punto de escucha, aunque se comprueba su uso para fijar la atención del espectador en el diálogo de los personajes y para determinar las relaciones espaciales entre los personajes y objetos de acuerdo con la acción narrativa, también se observó que este puede fijarse, por momentos, en la música y en los efectos de sonido.

Además de los efectos de sonido y ruidos empleados de forma diegética para dar verosimilitud a los relatos, destaca el uso creativo de sonidos y ruidos generados electroacústicamente en sustitución de sonidos 'reales' (el grito de Dios, truenos, ráfaga, agua, el sonido del shofar, que es más fuerte que todos los truenos).

En relación con los recursos técnicos, se encontró una riqueza en su variedad y combinación que potencian la expresividad del sonido para atraer la atención del espectador.

Entre las representaciones más vanguardistas de la voz de Dios en el cine, destaca la representación del personaje-voz mediante efectos de sonido y ruidos, música y silencio, sin recurrir a la voz humana para expresarse, así como la creación electroacústica de sonidos para representarla.

En suma, el lenguaje sonoro y las cualidades de los sistemas de signos que lo integran son imprescindibles para la construcción y credibilidad del mundo ficcional en cine; estos desempeñan funciones narrativas, que pueden adelantar o reforzar el relato visual, o contraponerse para generar diversos significados narrativos. Por ello, el modelo utilizado en el análisis del personaje-voz Dios es una herramienta que puede contribuir a pensar la voz de manera independiente a la palabra, y al análisis del lenguaje sonoro en el cine con categorías que privilegian sus cualidades acústicas.

\section{Referencias}

1. Arnheim, R. (1980). Estética radiofónica. Barcelona: Paidós.

2. Barthes, R. (1976). Listening. En The Responsibility of Forms (pp. 245-260). Nueva York: Hill and Wang.

3. Barthes, R. (2009). El grano de la voz. En Lo obvio y lo obtuso. Imágenes, gestos y voces (pp. 301-302). Barcelona: Paidós, Biblioteca Roland Barthes.

4. Chion, M. (2004). La voz en el cine. Madrid: Ediciones Cátedra.

5. Dolar, M. (2007). Una voz y nada más. Buenos Aires: Manantial.

6. Herman, D. (1999). Introduction. En Narratologies: new perspectives on narrative analysis (pp. 1-30). Columbus: Ohio State University Press.

7. Huwiler, E. (2005). Storytelling by sound: a theoretical frame for radio drama analysis. The Radio Journal International Studies in Broadcast and Audio Media, 3(1), 45-59. Doi: 10.1386/rajo.3.1.45/1

8. Huwiler, E. (2016). A narratology of audio art: telling stories by sound. En J. Mildorf \& T. Kinzel (Eds.), Audionarratology. Interfaces of sound and narrative. Berlín: De Gruyter. 


\section{DISERTACIONES}

ESTUDIOS

El papel del sonido en la comunicación: contribución, funciones y efectos

ISSN: $1856-9536$

Doi: http://dx.doi.org/10.12804/revistas.urosario.edu.co/disertaciones/a.6407

Volumen 12, Número 2 / Julio-diciembre 2019

Versión PDF para imprimir desde

http://revistas.urosario.edu.co/index.php/disertaciones

9. Klofstad C., Anderson R., \& Nowicki, S. (2015). Perceptions of competence, strength, and age influence voters to select leaders with lower-pitched voices. PLOS ONE, 10(8), e0133779. Doi: https://doi.org/10.1371/ journal.pone.0133779

10. Lacan, J. (2004). La voz de Yahvé. En Lacan, El seminario de Jacques Lacan (Libro 10, La angustia 1962-1963). Buenos Aires: Editorial Paidós. Recuperado de http://www.bibliopsi.org/docs/lacan/Seminario-10-La-Angustia-Paidos-BN.pdf

11. Larson, S. (2015). Pensar el sonido. Una introducción a la teoría y práctica del lenguaje sonoro cinematográfico. México: Universidad Nacional Autónoma de México-Centro Universitario de Estudios Cinematográficos.

12. Mildorf, J., \& Kinzel, T. (Eds.). (2016a). Audionarratology. Interfaces of sound and narrative. Berlín: De Gruyter.

13. Mildorf, J., \& Kinzel, T. (Eds.), (2016b). Multisensory imaginings: an audionarratological analysis of Philip Roth's novel indignation and its German radio play adaptation Empörung. Counter Text, 2(3), 307-321. Dol: 10.3366/ count.2016.0062. Recuperado de https://www.euppublishing.com/doi/full/10.3366/count.2016.0062

14. Rodero, E., \& Soengas, X. (2010). Ficción radiofónica. Madrid: Instituto Radio Televisión Española.

15. Rodero, E. (2005). Producción radiofónica. Madrid: Cátedra.

16. Schaeffer, P. (2003). Traité des objets musicaux. París: Seuil, 1966. [Tratado de los objetos musicales. Madrid: Alianza, 2003].

17. Zavala, L. (2010). El análisis cinematográfico y su diversidad metodológica. Revista Casa del Tiempo Cariátide, (30), 65-69. Recuperado de http://bit.ly/2DOLJyP

\section{Filmografía}

1. Adams, T., Caroselli, T., Milchan, A., Wachsberger, P. (productores) \& Edwards, B. (director). (1991). Switch [cinta cinematográfica]. eu: Motion Pictures Associates. eu: Beco Films, Cinema Plus, Home Box Office (нво).

2. Albela, M., Chernin, P., El Raie, M., Huffman, M., Schaefer, M., Ridley, S. (productores) \& Scott, R. (director). (2014). Exodus: gods and kings [cinta cinematográfica]. eu: Chernin Entertainment, Scott Free Productions, Babieka, Volcano Films.

3. Barber, G., Birnbaum, R., Bostick, M. (productores) \& Shadyac, T. (director). (2003). Bruce almighty [cinta cinematográfica]. Eu: Motion Pictures Associates. Eu: Spyglass Entertainment, Universal Pictures, Shady Acres Entertainment, Pit Bull Productions.

4. Brenner, R., Bright, S., Gill, M. (productores) \& Kassell, N. (director). (2011). A little bit of heaven [cinta cinematográfica]. eu: Davis Entertainment, Film Department, MJw.

5. DeMille, C. (productor) \& DeMille, C. (director). (1956). The ten commandments [cinta cinematográfica]. eu: Motion Pictures Associates.

6. Gladstone, E., Goldstein, J., Newall, P. (productores) \& Charles, L. (director). (2016) Army of one [cinta cinematográfica]. Eu: Conde Nast Entertainment, Dimension Films, Endgame Entertainment, Kasbah-Film Tanger.

7. Gordon, J., Greenlee, L., Mosier, S. (productores) \& Smith, K. (director). (1999). Dogma [cinta cinematográfica]. EU: View Asked Productions y sTK.

8. Katzenberg, J., Rabins, S., Finkelman, P. (productores) \& Chapman, B., Hickner, S., Wells, S. (directores). (1998). The prince of Egypt [cinta cinematográfica]. ev: DreamWorks Animation, DreamWorks. 


\section{DISERTACIONES}

ESTUDIOS

9. Messerli, W., Ruggiero, G., Schlossberg-Cohen (productores) \& Carr, J., Marshak, P., McGowan, T., Schlossberg-Cohen, J., Tallas, G. (directores). (1985). Night train to terror [cinta cinematográfica]. EU: Visto International Inc.

10. Parent, M., Milchan, A. (productores) \& Aronosfky, D. (director). (2014). Noah [cinta cinematográfica]. EU: Paramount Pictures, Regency Enterprises, Protozoa Pictures, Disruption Entertainment.

11. Tarkovski, A. (2009). Esculpir el tiempo. México: UNAM, Centro Universitario de Estudios Cinematográficos.

12. Warner, J., Wallis, H., Blancke, H. (productores) \& Connelly, M., Keighley, W. (directores). (1936). The green pastures [cinta cinematográfica]. eu: Warner Bros.

13. Weintraub, J. (productor) \& Reiner, C. (director). (1955). Oh, God! [cinta cinematográfica]. Eu: Motion Pictures Associates. Eu: Warner Bros. 